\title{
Nature de l'onde d'interface de Scholte sur une coque cylindrique
}

\author{
G. MAZE, F. LÉON, F. LECROQ, D. DÉCULTOT et H. ÜBERALL* \\ Laboratoire d'Acoustique Ultrasonore et d'Electronique, LAUE, URA 1373 du CNRS, Place Robert \\ Schuman, 76610 Le Havre, France \\ ${ }^{*}$ Physics Department, Catholic University of America, Washington, DC 20064, U.S.A.
}

\begin{abstract}
The theoretical and experimental studies of the ultrasonic scattering by cylindrical shells filled with air and immersed in water show, on the backscattering spectra, large amplitude variations. These variations are related to an interface wave called "A wave". The resonances of this wave are detected in a frequential window that depends on the radius ratio (inner radius b/outer radius a). They are observed for cylindrical shells with a radius ratio superior to 0.6. This wave that propagates in the water around the pipe is still not very well know in spite of the investigations which have been devoted to it. In this numerical study, it is proposed a study of the evolution of the frequency of different resonances for cylindrical shells with the radius ratio between 0.2 and 0.95 . The interaction between the flexural wave $A_{0}$ in the shell and the creeping wave in the fluid around the pipe is studied in greater detail. The dispersion curves of the phase velocity for these waves are presented. They show a repulse area between these two curves. This area is translated toward the high frequencies when the radius ratio tends toward 1 . This phenomenon is related to the contact condition at the liquid/shell interface.
\end{abstract}

\section{INTRODUCTION}

Les fonctions de forme [1,2], obtenues à partir de la diffusion d'une onde acoustique plane par un tube élastique rempli d'air et plongé dans un liquide, montrent la présence de grandes variations d'amplitude qui sont attribuées à des résonances liées à une onde d'interface appelée onde de Scholte ou onde $\mathrm{A}[3,4]$. L'influence des résonances dues à cette onde se fait sentir lorsque le rapport des rayons b/a (b: rayon interne, a: rayon externe) est supérieur à 0,6 . Ces résonances sont observables dans une fenêtre fréquentielle qui glisse vers les hautes fréquences lorsque le rapport des rayons tend vers 1 . Il est montré, pour conforter la nature de ce type d'onde, que la vitesse de phase, calculée à partir des modes de résonances, ne dépasse jamais la vitesse de propagation des sons dans le liquide entourant le tube. L'essentiel de l'énergie vibratoire se situe dans le fluide comme pour l'onde de Scholte rencontrée sur une interface plane. Il faut noter, toutefois, une différence importante entre la propagation sur une interface plane (il n'existe pas de conversion de cette onde en onde de volume) et la propagation sur l'interface courbe d'un tube (il existe une conversion qui est fonction de la fréquence). Certaines études numériques [6], effectuées à partir d'une coque sphérique fine immergée dans un liquide, montrent la présence de deux courbes de dispersion de vitesse de phase qui peuvent être attribuées à l'onde $\mathbf{A}_{\mathbf{0}}$ en contact avec un fluide. Les deux ondes, correspondant aux courbes de dispersion, ont été appelées $\mathbf{a}_{0+}$ et $\mathbf{a}_{0-}$. Les auteurs de cette référence parlent de "bifurcation" de l'onde $\mathbf{A}_{0}$ lorsqu'elle est en contact avec un fluide. Dans ce travail, la vitesse de phase de cette onde est étudiée systématiquement en fonction de la fréquence pour différentes valeurs du rapport $\mathbf{b} / \mathbf{a}$. 


\section{ONDES DE FLEXION SUR UNE COQUE CYLINDRIQUE}

Une coque cylindrique circulaire en acier inoxydable $\left(\rho_{2}=7900 \mathrm{~kg} / \mathrm{m}^{3}, c_{L}=5790 \mathrm{~m} / \mathrm{s}, \mathbf{c}_{\mathbf{T}}=3100 \mathrm{~m} / \mathrm{s}\right)$ de longueur infinie, immergée dans de l'eau $\left(\rho_{1}=1000 \mathrm{~kg} / \mathrm{m}^{3}, c_{1}=1470 \mathrm{~m} / \mathrm{s}\right)$ et dont la cavité est vide, est insonée par une onde plane sous un angle d'incidence $\alpha$ par rapport à la normale à son axe. Le problème de diffusion conduit à la résolution d'un système linéaire de sept équations à sept inconnues qui peut se mettre sous la forme: $\left[\mathbf{D}_{\mathbf{n}}\right] \cdot\left[\mathbf{B}_{\mathbf{n}}\right]=\left[\mathbf{C}_{\mathbf{n}}\right](\mathbf{1})$, avec $\left[\mathbf{D}_{\mathbf{n}}\right]$ une matrice $7 \times 7,\left[\mathbf{B}_{\mathbf{n}}\right]$ le vecteur colonne des inconnues et $\left[\mathbf{C}_{\mathbf{n}}\right]$ le vecteur colonne du second membre [7]. Dans tout ce qui suit, la direction dincidence sera considérée comme normale $(\alpha=0)$. Le déterminant $\mathbf{D}_{\mathbf{n}}$ s'écrit alors:

$$
D_{n}=x_{1} H_{n}(1)^{\prime}\left(x_{1}\right) \Delta_{n}^{(11)}-\rho-\frac{x T^{2}}{2} H_{n}\left(x_{1}\right) \Delta_{n}^{(12)}
$$

$x_{1}$ est la fréquence réduite $\left(x_{1}=k_{1} a\right), x_{T}$ peut se déduire de la relation $x_{T}=x_{1} c_{1} / c_{T}, \rho$ est le rapport des masses volumiques $\rho_{1} / \rho_{2}, \Delta_{n}{ }^{(11)}$ et $\Delta_{n}{ }^{(12)}$ sont des mineurs d'ordre 6 . Le premier mineur peut se décomposer en un produit de deux sous-déterminants: $\Delta_{n}{ }^{11}=\Delta_{n}(\mathcal{C}) \cdot \Delta_{n}{ }^{(g)}$ (3). $\Delta_{n}{ }^{(C)}$ est un sousdéterminant $4 \times 4$ dont les racines sont les modes propres liés aux ondes circonférentielles et $\Delta_{\mathbf{n}}{ }^{(\mathrm{g})}$ est un sous-déterminant $2 \times 2$ dont les racines sont les modes propres liés aux ondes guidées. Le second sousdéterminant est présent à cause de la méthode de calcul. Il faut remarquer ici que ce sous-déterminant n'interviendra pas par la suite dans les calculs. Les résonances qui sont mises en évidence sur le spectre de rétrodiffusion sont liées aux racines complexes du déterminant $\mathbf{D}_{\mathbf{n}}$ qui peuvent se mettre sous la forme: $\underline{x}_{n 1}=x_{n 1}+i \Gamma_{n} / 2$ (4), où $x_{n 1}$ est la fréquence réduite de la résonance et $\Gamma_{n}$ est la largeur de la résonance. Cette dernière est liée au pouvoir de réémission de l'onde circonférentielle ou guidée dans le fluide. La partie réelle de la racine complexe (4) correspond à la fréquence réduite du mode propre du tube placé dans le vide pour les résonances qui sont expérimentalement observables. Ces modes propres sont associables, soit à des ondes circonférentielles, soit à des ondes guidées dans la coque. Le facteur multiplicatif qui se trouve devant le sous-déterminant $\Delta_{\mathbf{n}}{ }^{11}$ est la dérivée première de la fonction de Hankel de première espèce. Les racines de cette dérivée sont des modes qui sont liés aux ondes de Franz. Ces ondes, encore appelées ondes rampantes, se propagent autour d'un cylindre parfaitement rigide. Le second terme de l'équation (2) permet d'obtenir les modes propres d'un tube "mou".

\section{ANALYSE DES RÉSULTATS}

Le rapport des rayons b/a des tubes est compris entre 0,2 et 0,95. Les courbes (fig. 1) montrent l'évolution de la partie réelle $\left(x_{n 1}\right)$ et de la partie imaginaire $\left(\Gamma_{n} / 2\right)$ des modes $n=3$ et 10 du déterminant (2) en fonction du rapport des rayons b/a. Ces courbes montrent qu'il existe deux types de résultats. Dans le cas des modes $(n=2-4)$, les courbes donnant la partie réelle en fonction du rapport des rayons se croisent alors que celles donnant la partie imaginaire ne se croisent pas. Lorsque les modes sont supérieurs à 4 , c'est le contraire. Dans le premier cas, les courbes (†) sont à mettre en relation avec une onde rampante appelée Stoneley lorsque le cylindre est massif et élastique. Cette onde a des caractéristiques voisines de celles de l'onde de Franz 1 sur un cylindre rigide [8]. Les courbes $(\boldsymbol{D})$ sont à mettre en relation avec une onde se propageant dans la coque. Elle est appelée $\mathbf{A}_{0}$ lorsqu'elle peut être assimilée à la première onde de Lamb antisymétrique sur une plaque. Dans le second cas $(n>4)$, les remarques faites précédemment s'appliquent pour la partie gauche des courbes, en dehors de la zone d'interaction. Connaissant le mode, il est possible de calculer la vitesse de phase des ondes circonférentielles associées à l'aide de la relation:

$$
\mathbf{C p h}=\mathrm{C}_{1} \quad \mathrm{x}_{\mathrm{n1}} / \mathrm{n} \quad(5) \text {, }
$$

Sur la figure 2 sont tracées les courbes de dispersion de la vitesse de phase des ondes circonférentielles pour des tubes dont le rapport des rayons est respectivement égal à 0,2 (A) et 0,9 (B). Dans le cas du rapport 0,9 , ces deux courbes seront désignées par $\mathbf{A}_{0}{ }^{+}$pour celle qui se trouve au-dessus et par $\mathbf{A}_{0}{ }^{-}$pour celle du dessous. Ces notations sont introduites en accord avec celles définies dans la référence [6] pour des coques fines sphériques $\left(a_{0+}, a_{0-}\right)$. Les courbes de dispersion des vitesses de phase de l'onde de Franz 
$\left(F_{1}\right)$ autour d'un cylindre rigide sont reportées $(\Delta)$ ainsi que les courbes de dispersion de l'onde circonférentielle $\mathbf{A}_{0}$ d'un tube dans le vide calculée à partir du déterminant (3) (3). Dans le cas d'un tube de rapport des rayons $\mathbf{b} / \mathbf{a}=\mathbf{0}, \mathbf{2}$ (fig. $2 \mathrm{~A}$ ), les courbes de dispersion calculées à partir de l'équation (2) se confondent pratiquement avec les courbes calculées à partir de la dérivée de la fonction de Hankel d'une part et du déterminant (3) d'autre part. Il est donc normal de dire que les ondes, qui sont générées dans et autour de ce tube plongé dans l'eau, sont l'onde circonférentielle $\left(\mathbf{A}_{\mathbf{0}}\right)$ et l'onde de Stoneley décrite dans la référence [8]. Pour ce tube $(\mathbf{b} / \mathbf{a}=\mathbf{0 , 2} ;$ fig.2A), les courbes de dispersion sont bien séparées. Les propagations acoustiques dans le tube et dans l'eau sont pratiquement indépendantes. Dans ce cas, il faut noter que les parties imaginaires des racines complexes (Fig.3A) sont grandes dans toute la bande de fréquence. Cela entraîne des difficultés pour les mettre en évidence expérimentalement sous la forme de pics de résonances. Cette dernière remarque est surtout vraie pour l'onde Stoneley (E). Pour les tubes dont le rapport des rayons est supérieur à 0,7 (fig. $2 \mathrm{~B}, \mathbf{b} / \mathbf{a}=0,9$ ), l'interaction se fait nettement sentir dans un domaine de fréquence qui croît avec le rapport $\mathbf{b} / \mathbf{a}$. Cette interaction est liée au fait que la vitesse de phase de l'onde $\mathbf{A}_{0}$ diminue pour une même fréquence lorsque $\mathbf{b} / \mathbf{a}$ tend vers 1 . Comme la courbe de dispersion de l'onde $\left(F_{1}, \Delta\right)$ du cylindre rigide est insensible à l'épaisseur de la coque, les deux courbes vont se couper. $\mathrm{La}$ présence de l'eau fait que, dans la région du croisement potentiel, les courbes de dispersion liées au tube élastique se séparent. Les ondes ne peuvent plus être assimilées à l'onde circonférentielle $\left(\mathbf{A}_{0}\right)$ d'une part et à l'onde rampante de Franz d'autre part. En suivant la courbe de dispersion supérieure, la vitesse de phase est voisine de celle de l'onde de Franz 1 rigide en basses fréquences alors qu'elle est identique à celle de l'onde circonférentielle $\left(\mathbf{A}_{\mathbf{0}}, \boldsymbol{t}\right)$ en hautes fréquences et vice-versa pour la courbe inférieure. Il faut noter que ce phénomène se traduit sur la partie imaginaire des racines du déterminant (2) par des variations très importantes. Les courbes $\Gamma / 2\left(\mathbf{x}_{1}\right)$ (fig.3B) se croisent. En basse fréquence, il faut noter qu'une branche correspond à une partie imaginaire petite qui sousentend la possibilité de détection des modes. Cette branche, qui correspond à la courbe de dispersion de $\mathbf{A}_{\mathbf{0}}$, est traditionnellement associée à l'onde $\mathbf{A}$.

\section{CONCLUSION}

Cette étude tente de comprendre le mécanisme physique qui explique la présence de résonances dans un domaine de fréquence limité qui se translate vers les hautes fréquences lorsque le rapport des rayons b/a croît. Ces résonances peuvent être attribuées à l'onde que nous avons désignée par $\mathbf{A}_{0}{ }^{-}$et qui est traditionnellement appelée onde de Scholte ou $\mathbf{A}$ dans la fenêtre de fréquence où elle est détectable. Cette onde est due à l'interaction de l'onde $\mathbf{A}_{0}$ avec une onde rampante proche de l'onde de Franz 1 sur un cylindre rigide. Cette interaction est maximale lorsque la courbe de dispersion de l'onde de Franz autour d'un tube rigide coupe la courbe de dispersion de l'onde circonférentielle $\mathbf{A}_{0}$ dans un tube placé dans le vide. Cette interaction entraîne l'existence d'un coefficient de réémission petit pour une fréquence inférieure à celle du croisement. En outre, la fréquence du croisement se translate vers les hautes fréquences lorsque le rapport des rayons tend vers 1 . Ces deux dernières remarques expliquent pourquoi les résonances liées à cette onde sont visibles dans une fenêtre fréquentielle qui glisse vers les hautes fréquences lorsque le rapport des rayons b/a tend vers 1 . La dénomination d'onde de Scholte, qui est attribuée à cette onde, ne doit pas créer une confusion avec l'onde de Scholte sur une interface plane. Cette onde ne peut se concevoir que par l'interaction entre onde rampante et onde circonférentielle dans la coque.

[1] Gérard A., Rousselot J.L., Izbicki J.L., Maze G. \& Ripoche J., Rev. Phys. Appl. 23 (1988) 289-299.

[2] Kaplunov J.D., Veksler N.D., Acustica 72 (1990) 131-139.

[3] Rousselot J.L., Acustica 58 (1985) 1291-297.

[4] Izbicki J.L., Maze G. \& Ripoche J., Acustica 61 (1986) 137-139.

[5] Izbicki J.L., Rousselot J.L., Gérard A., Maze G. \& Ripoche J., J. Acoust. Soc. Am. 90 (1991) 26022608.

[6] Sammelmann G.S., Trivett D.H. \& Hackman R.H., J. Acoust. Soc. Am. 85 (1989) 114-124.

[7] Léon F., Lecroq F., Décultot D. \& Maze G., J. Acoust. Soc. Am. 91 (1992) 1388-1397.

[8] Clotteau B., Conoir J.M., Rousselot J-L \& Derem A., J. Acoustique. 3 (1990) 213-242. 

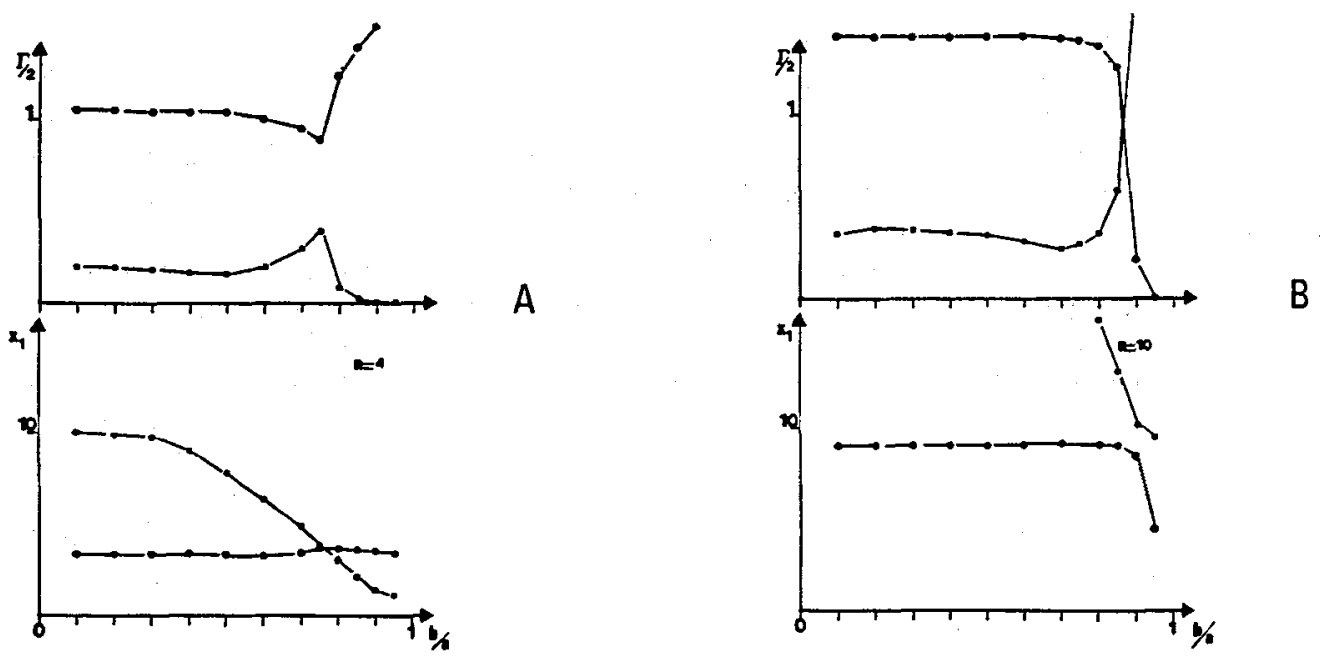

Fig.1: Courbes donnant l'évolution de la fréquence réduite $x_{1}$ et de la largeur $\Gamma / 2$ d'un mode $n$ en fonction du rapport des rayons b/a.
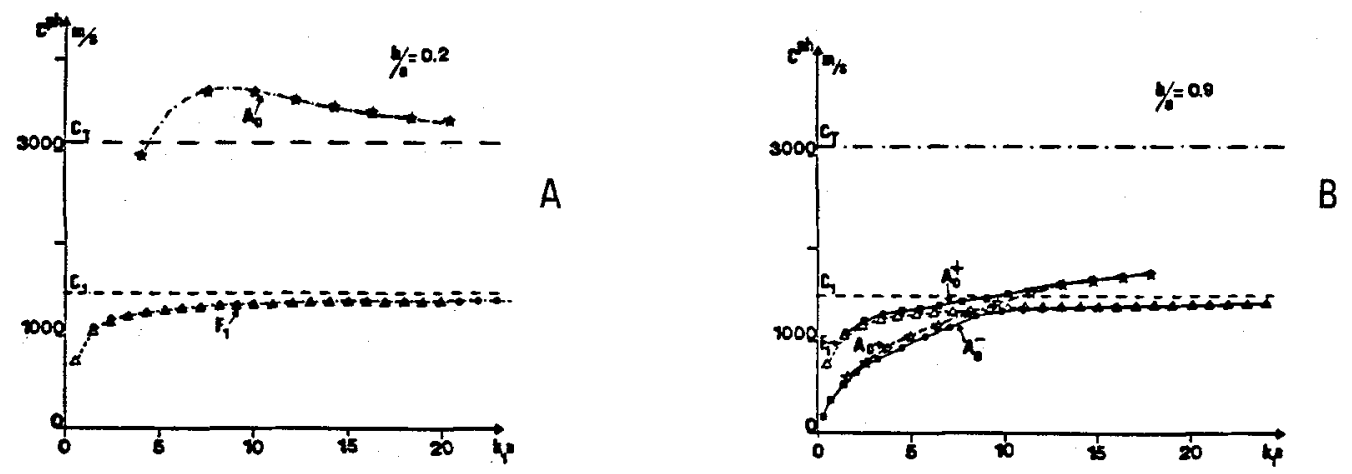

Fig.2: Courbes de dispersion des vitesses de phase pour deux tubes différents; $A: b / a=0,2 ; B: b / a=0,9$.
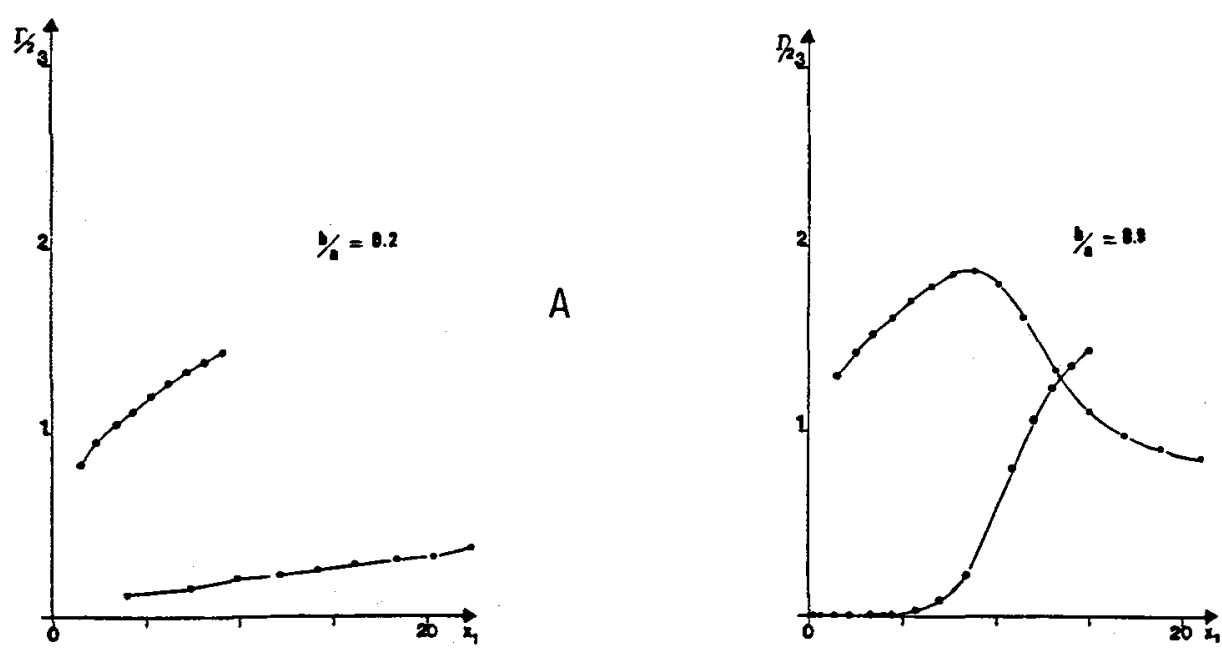

Fig.3: Courbes donnant la largeur d'une résonance en fonction de la fréquence réduite pour deux tubes différents; $A: b / a=0,2 ; B: b / a=0,9$. 